\title{
Free Carrier Distribution Criterion in Quantum Dot Lasers
}

\author{
HIFSA SHAHID*
}

RECEIVED ON 05.03.2015 ACCEPTED ON 28.05.2015

\begin{abstract}
The spontaneous emission spectra of a 1.28 $\mu \mathrm{m}$ InAs/GaAs QD (Quantum Dot) Fabry-Pérot laser device has been measured under continuous wave operation at a fixed junction temperature of $300 \mathrm{~K}$. At low carrier densities, empirically observed static peak wavelength position and a fixed spectral shape of the spontaneous emission spectra are indicative of the random-like population distribution rather than a global Fermi level in the system. A theoretical model based on the Monte-Carlo method has been shown to have good agreement with the empirical results. In addition the evolutions of spontaneous emission spectral shapes are also explained in terms of many body effects.
\end{abstract}

Key Words: Quantum Dot Laser, Spontaneous Emission, Gain, Thermal.

\section{INTRODUCTION}

$\mathrm{T}$

he InAs/GaAs based QD lasers are currently being commercialized [1] for FTTH (Fiber to the Home) applications. In order to optimize the device performance, the effect of free carrier distribution among dots and many body effects in a QD laser device are of crucial importance to understand. These can play a major role in the development of new high-speed modulation schemes [2-3] and in improving the dynamic characteristics of a QD laser device.

Until now different techniques are employed to access the carrier distribution trends among QD. In the past the measurements such as spontaneous emission, gain and current vs. I-V (Voltage) characteristics have been used to access the carrier distribution among dots as a function of average number of carriers per dot/current density. It has been reported earlier [4-7] that the carrier distribution among the dot follows both Fermi-Dirac and random-like trends which are also sometimes, regarded as thermal and non-thermal distributions respectively.

Random-like distribution has an advantage in terms of possibility of getting superior modulation characteristics such as mode-locking [8-10]. However, thermalized way of carrier distribution shows the dominance of self-heating effects in a quantum dot laser device. So in this case, the carrier distribution would be thermally driven, affecting directly the performance of a QD laser device.

QDs are expected to have strong carrier localization because of their 3D confinement showing atom like properties, with their s and p orbitals, exhibit the discrete

* Assistant Professor, Department of Electrical Engineering, University of Engineering \& Technology, Kala Shah Kakoo Campus, Lahore. 
nature of their ground and excited states. Ideally, the QDs of the same size (inhomogeneous line width $\sim 0$ ), isolated from each other without wetting layer [11] and with state separation more than thermal activation energy (kT $=26 \mathrm{meV}$ at $300 \mathrm{~K}$ in InAs/GaAs dots) are suggestive of exhibiting a random carrier distribution trend. In this case, as is theoretically expected that the spectral shapes of the luminescence intensity from each dot and peak intensity wavelength for each of the radiative state would remain unchanged. Hence, would show an independent behavior from an increase in temperature or current density/carriers per dot. However, it is practically observed that there is a non-ideal carrier localization in dots and the carrier transfer can take place at either elevated temperatures or at high current densities via wetting layer. In this case, the QD system exhibits a global Fermi level losing its identity and properties. In order to minimize the effects of self heating on performance, development of devices with as high a value of ' $\mathrm{T}_{\mathrm{o}}$ ' as could be achieved has been suggested [12].

This paper presents an empirical analysis and then a theoretical model to describe the carrier distribution as a function of current density among dots. For the purpose, spontaneous emission characteristics of a $1.28 \mu \mathrm{m}$, InAs/ GaAsFabry-Pérot QD laser device are derived and analyzed at a fixed junction temperature of $300 \mathrm{~K}$. Therefore, accompanying self-heating effects are kept fixed at each of the increased current density levels under consideration. An, important information about the spectral shapes due to many body effects and peaks of the spontaneous emission spectra is retrieved as a function of increased current density steps to access the carrier distribution trends among the dots.

\section{DEVICE STRUCTURE FABRICATION}

AND

The QD laser device used for the analysis is grown by Innolume via MBE (Molecular Beam Epitaxy) technique.
It is a $1.28 \mu \mathrm{m}$ InAs/GaAsFabryPèrot QD laser device comprising of ten QD layers with an aerial dot density of $4 \times 10^{10} / \mathrm{cm}^{2}$. The device structure and fabrication details are shown in Fig. 1 and are explained elsewhere [13]. It is an edge emitting laser device. The laser bars are "as cleaved" and facet reflectivity is achieved as 33\%. For the analysis, such shorter lengths are cleaved for which it was made sure to keep the mirror loss higher than the peak gain of the laser material and non-lasing characteristics were achieved. Therefore, it was made possible to analyze the laser device at elevated current densities without lasing. The junction temperature is kept fixed [13] and therefore free carrier distribution is analyzed without self-heating effects.

\section{EXPERIMENTAL SET UP AND FIXED JUNCTION TEMPERATURE CONDITION}

For high-resolution spectroscopy, the experimental setup is shown in Fig. 2. In this case a $10 \mathrm{pm}$ resolution was adjusted to resolve the electroluminescence spectrum. The analysis is performed under continuous wave mode and the junction temperature was kept fixed at $300 \mathrm{~K}$. A methodology to eliminate the accompanied increasing self heating effects at elevated current densities is described elsewhere [14]. Then it was made possible to analyze carrier distribution solely at a fixed temperature without any influence of the self heating effects.

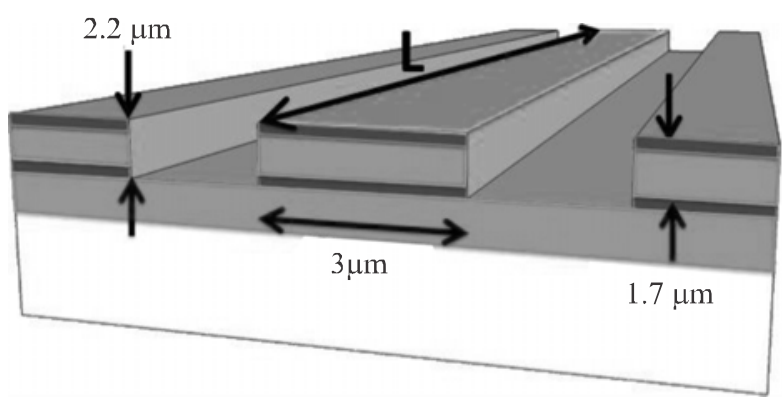

FIG. 1. QD LASER DEVICE STRUCTURE 


\section{QUANTUM DOT LASER DEVICE: OPTICAL POWER CHARACTERISTICS}

A non lasing QD laser device selected for the analysis is $300 \times 3 \mu \mathrm{m}$. The continuous wave, device characteristics i.e. optical power as a function of current density at $300 \mathrm{~K}$ heat sink temperature is shown in Fig. 3. The device indicates saturation above $6 \mathrm{kA} / \mathrm{cm}^{2}$. Therefore, the analysis was performed up to $\sim 5 \mathrm{kA} / \mathrm{cm}^{2}$.

\section{SPONTANEOUS EMISSION CHARACTERISTICS}

At the initial stage, in order to derive the spontaneous emission spectra, the electroluminescence spectra are retrieved as a function of current density. A spectrum at $4 \mathrm{kA} / \mathrm{cm}^{2}$ is shown in Fig. 4. The single mode behavior of the device is confirmed via inspection of the spectrum at each of the current density steps, as mentioned in the insets of Fig. 4.

Via using Equation (1); the Hakki and Paoli method [15] the spontaneous emission spectra are derived at each wavelength of the electroluminescence spectrum.

Spontaneons Emission $=\frac{4 \mathrm{P}_{\mathrm{i}} \mathrm{V}_{\mathrm{i}}}{\left(\sqrt{\mathrm{P}_{\mathrm{i}}}+\sqrt{\mathrm{V}_{\mathrm{i}}}\right)^{2}}$

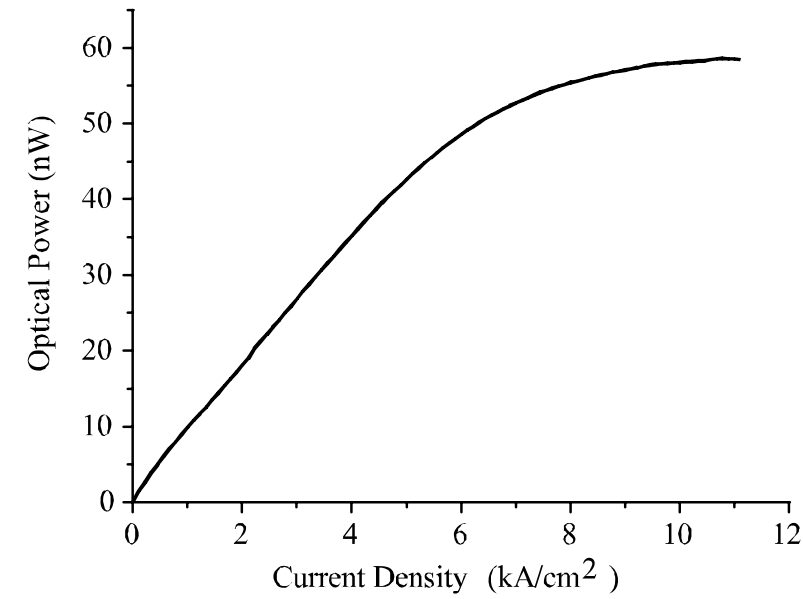

FIG. 3. OPTICAL POWER VERSUS CURRENT DENSITY FOR $300 \times 3 \mu \mathrm{m}$ AT 300K HEAT SINK TEMPERATURE

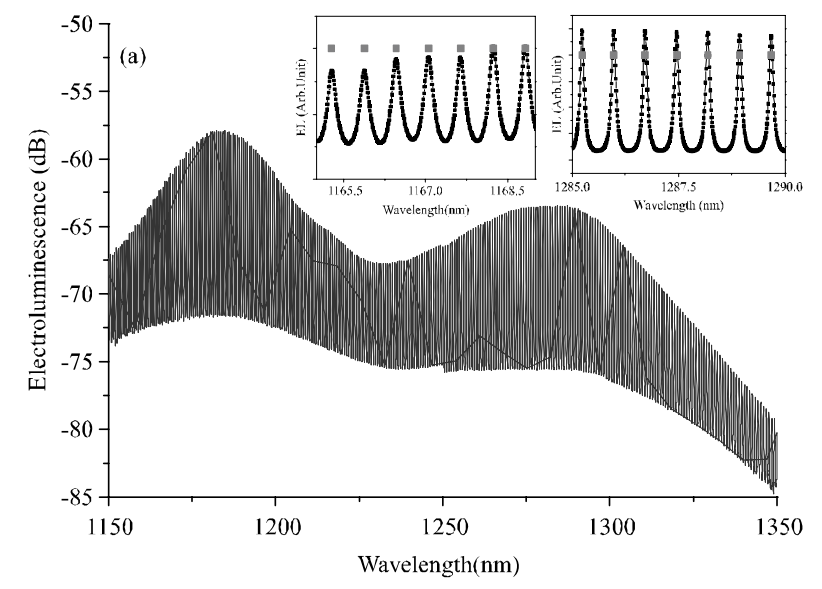

FIG. 4. THE ELECTROLUMINESCENCE SPECTRUM AS A FUNCTION OF WAVELENGTH FOR 300x3 $\mu$ M (LOWER CASE M), QUANTUM DOT LASER DEVICE. INSETS: PARTS OF THE LECTROLUMINESCENCE SPECTRUM SHOWN IN FIG. 3, CONFIRM THE SINGLE MODE BEHAVIOUR OF THE LASER DEVICE

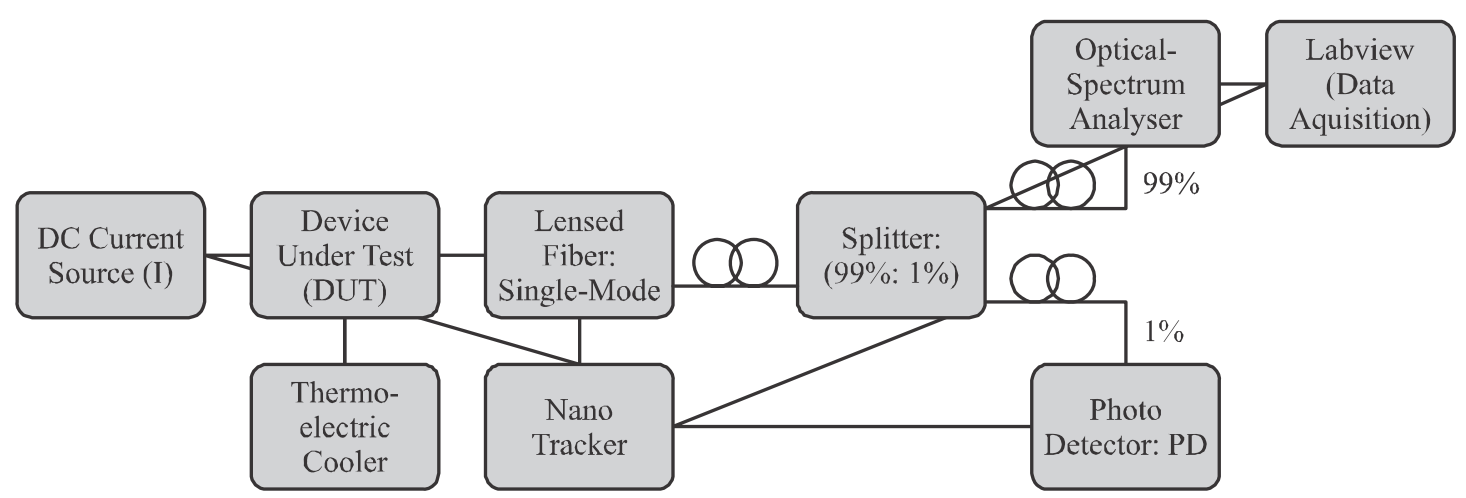

FIG. 2. HIGH RESOLUTION SPECTROSCOPY EXPERIMENTAL SET UP

Mehran University Research Journal of Engineering \& Technology, Volume 35, No. 3, July, 2016 [p-ISSN: 0254-7821, e-ISSN: 2413-7219] 
In Equation (1), $\mathrm{P}_{\mathrm{i}}$ and $\mathrm{V}_{\mathrm{i}}$ correspond to the peak and valley of an electroluminescence spectrum as shown in Fig. 5.

At the initial stage, in order to derive the spontaneous emission spectra, the electroluminescence spectra are retrieved as a function of current density. A spectrum at $4 \mathrm{kA} / \mathrm{cm}^{2}$ is shown in Fig. 3. The single mode behavior of the device is confirmed via inspection of the spectrum at each of the current density steps, as mentioned in the insets of Fig. 4.

The Fig. 6(a) plots the ground state normalized spontaneous emission spectral data vs. wavelength as a function of current density at a fixed junction temperature of $300 \mathrm{~K}$ for $1.28 \mu \mathrm{m}$ quantum dot Innolume material under continuous wave operating condition.

Now, the carrier distributions can be investigated at fixed heating effects. In this case, peaks of ground and excited states of the spontaneous emission spectra and their corresponding spectral shapes would indicate the carrier distribution among dots.

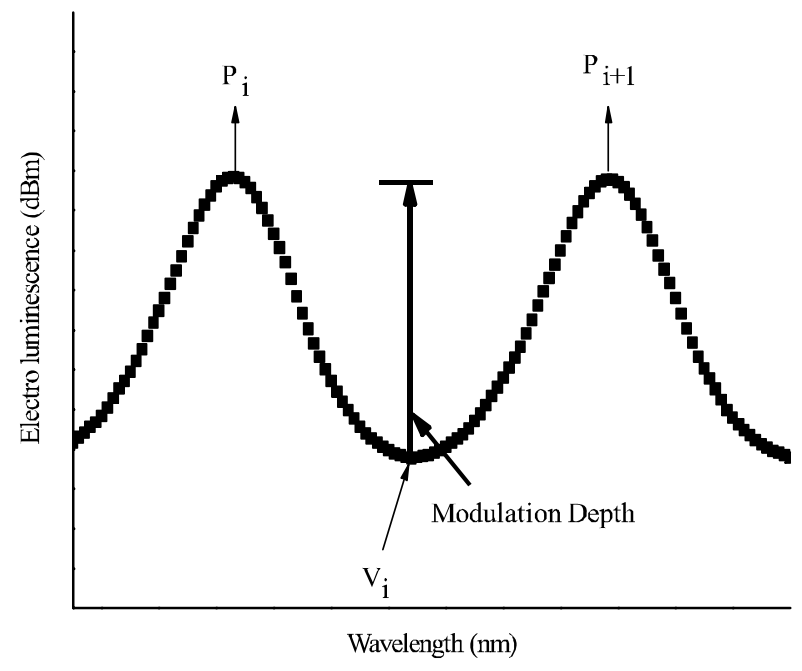

FIG. 5. THE ELECTROLUMINESCENCE FROM THE LASER DEVICE UNDER TEST INDICATING PEAKS (Pi) AND VALLEY (Vi).
As the, state separation is $(\sim 70 \mathrm{meV})$ more than the thermal activation energy, therefore the thermalized carrier distribution is expected to get minimized in this case.

From Fig. 6(b) it can be observed that from $44 \mathrm{~A} / \mathrm{cm}^{2}(\sim 0.03$ carriers/dot) to $222 \mathrm{~A} / \mathrm{cm}^{2}$ ( $\sim 0.1$ carriers/dot), the ground state peak and spectral shapes do not change as a function of current density and the FWHM (Full Width Half Maximum) also remains unchanged with a variation within the limits of $\sim \pm 1 \mathrm{meV}$. These observations point towards the realization that for the current density range under consideration, variation in homogeneous linewidth of the ground state QDs ensemble is least and therefore the behavior of quantum dot ensemble can be regarded as non-interacting isolated dots. Furthermore, the results indicate that the relative carrier density among dots does not change, remains independent of overall injection carrier density with no global Fermi level observed. Therefore it can be argued that these characteristics exhibit a random nature of free carrier distribution trend at these low dot occupancies.

After ground state saturation an evident red shift and reshaping of the spectral shapes is again suggestive of random distribution following the Poisson statistics and the many body effects i.e. band gap shrinkage and renormalization [16].

After ground state saturation an evident red shift and reshaping of the spectral shapes is again suggestive of random distribution following the Poisson statistics and the many body effects i.e. band gap shrinkage and renormalization [16].

\section{COMAPRISON: MODELLING WITH EMPIRICAL RESULTS}

The modeling results as shown in Figs. 7(a-b) and Fig. $8(\mathrm{a}-\mathrm{b})$ are plotted by using "Monte Carlo" method, and 
do not include self-heating and free carrier effects. The trends and comparison with the experimental results can be used to verify the carrier distribution criterion among dots.

From Fig. 8(a-b) which are derived from Fig. 7(a-b) it can be observed that for Fermi distribution case, the ground state and excited state exhibit a continuous blue shift as a function of average carriers per dot.

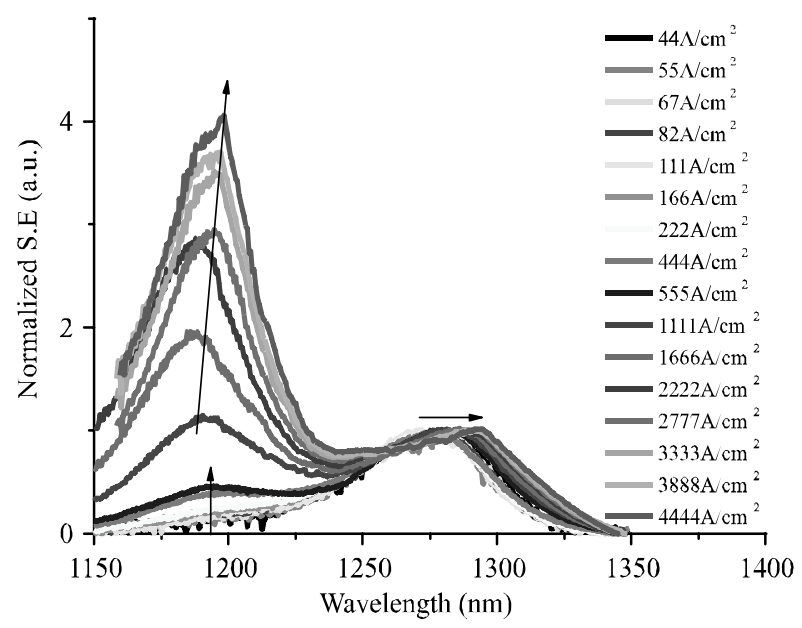

FIG. 6(a). GROUND STATE NORMALIZED SPONTANEOUS EMISSION SPECTRA AS FUNCTION OF WAVELENGTH AT 30OK CONSTANT JUNCTION TEMPERATURE FOR $1.28 \mu \mathrm{m}$ INNOLUME QUANTUM DOT LASER MATERIAL

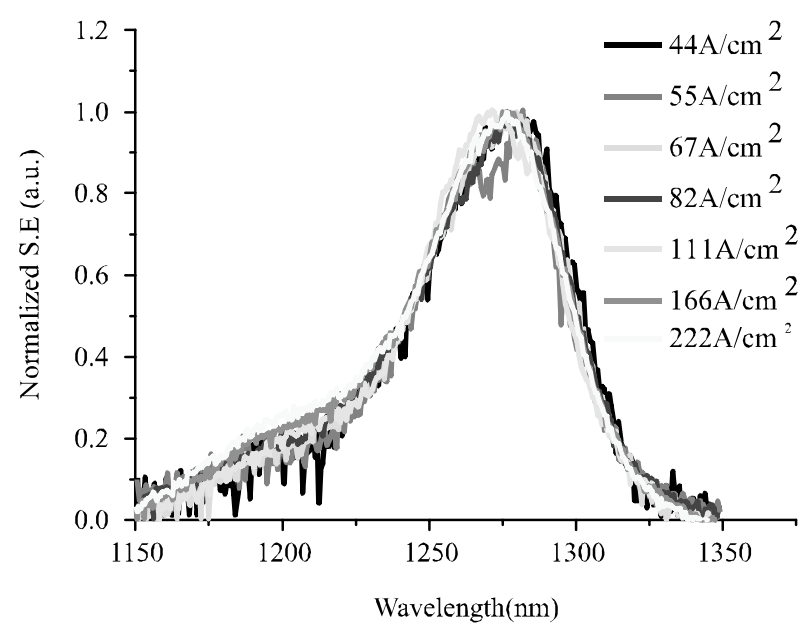

FIG. 6(b). NORMALIZED SPONTANEOUS EMISSION DATA FOR THE CURRENT DENSITY RANGE SHOWING FWHM REMAINS CONSTANT
However for random distribution case a different trend is observable for both states showing no shift in their peak spectral positions. However, at high current densities show a continuous red shift.

In order to compare the experimental data with the modeling results we need to look at the peak wavelength positions and spectral shapes of the states up to $\sim 2$ carriers per dot i.e. the ground state saturation.

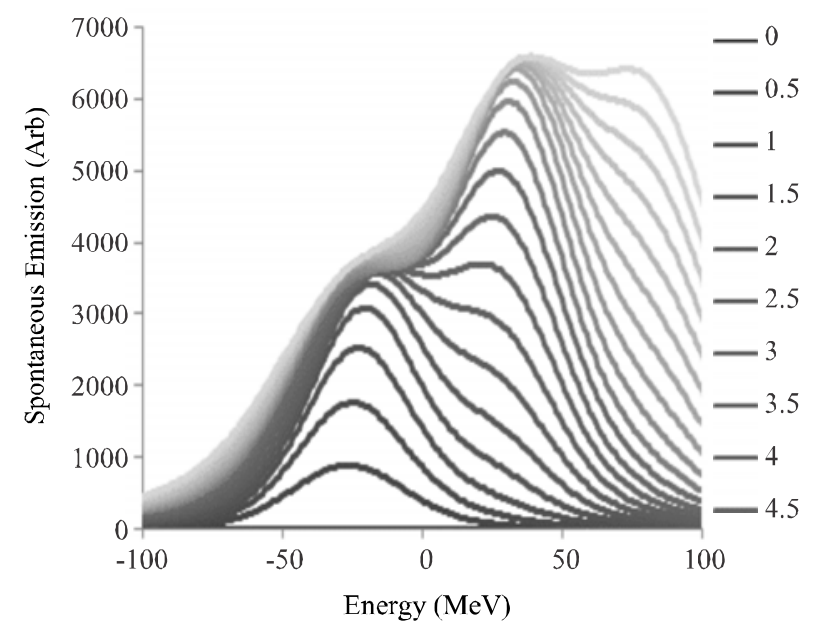

FIG. 7(a). SPONTANEOUS EMISSION AS A FUNCTION OF ENERGY SHOWING THE CARRIER DISTRIBUTION FOLLOWING THE FERMI-DIRAC STATISTICS

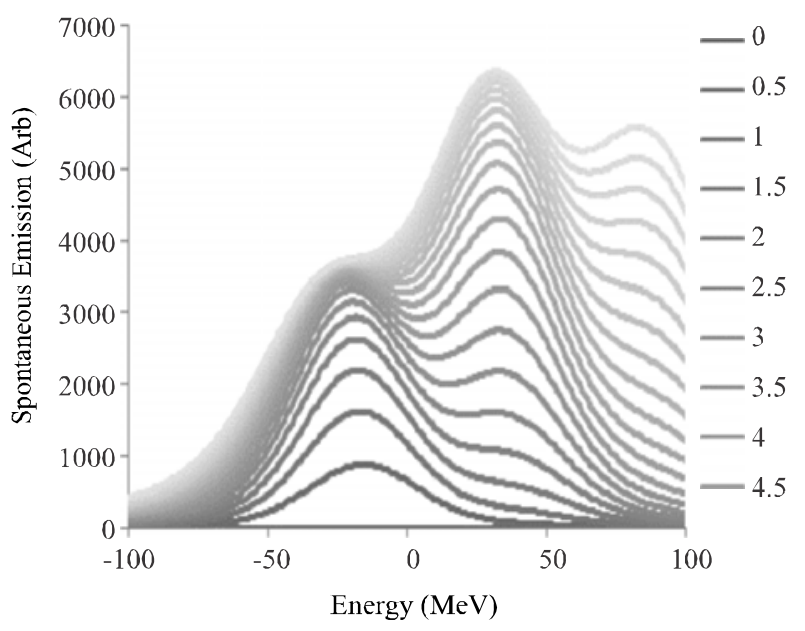

FIG. 7(b). SPONTANEOUS EMISSION AS A FUNCTION OF ENERGY SHOWING THE RANDOM NATURE OF CARRIER DISTRIBUTION

Mehran University Research Journal of Engineering \& Technology, Volume 35, No. 3, July, 2016 [p-ISSN: 0254-7821, e-ISSN: 2413-7219] 
The experimental results show that the ground state peak position does not shift up to $\sim 0.1$ carriers/dot but after that starts showing a continuous red shift which agrees with the modeling results indicating the random distribution of the carriers among dots.

Regarding the spectral shape comparison, up to $\sim 0.1$ carriers/dot, the experimental and the modeled data show similar results and spectra evolve

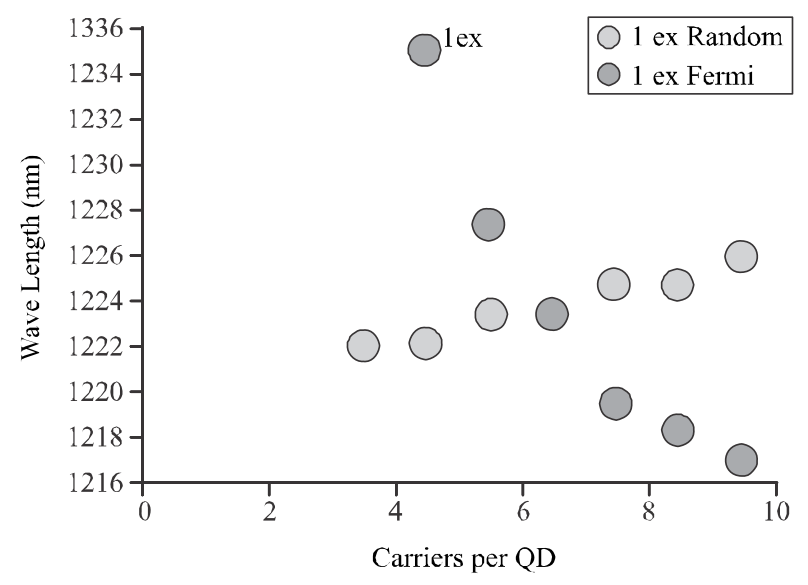

FIG. 8(a). EXCITED STATE PEAK WAVELENGTH SHIFT AS A FUNCTION OF AVERAGE CARRIERS PER DOT FOR RANDOM AND FERMI CARRIER DISTRIBUTION



FIG. 8(b). GROUND STATE PEAK WAVELENGTH SHIFT AS A FUNCTION OF AVERAGE CARRIERS PER DOT FOR RANDOM AND FERMI CARRIER DISTRIBUTION independently of the current densities. However, after that an asymmetric reshaping (band gap renormalization due to many body effects [17-18]) is observed in case of the experimental results. The similar behavior is not observed for the case of the modeled spectra which shows random carrier distribution criterion.

This is due to the fact that the modeled data presented in the paper does not include free carrier (many body effects) effects. Resultantly, on the basis of analysis and comparison presented here, before ground state saturation ( $\sim 2$ e-h pairs $)$ the experimental data shows that the carrier distribution among the dots for ground state follows the random distribution trend. The same observation for the excited state is again suggestive of random distribution of the carriers among dots. Therefore, over all behavior of the laser device indicates the random nature of carrier distribution among dots when heating effects are not appreciable.

\section{CONCLUSION}

This paper discussed the significance and presented a methodology to determine the carrier distribution criterion among dots of a commercial 1.28 $\mu \mathrm{m} \mathrm{GaAs} /$ InAs QD laser device, while maintaining the junction temperature at a fixed value of interest to be used for FTTH application. In this case, carrier distribution was made entirely independent of accompanied self heating effects for the current densities of interest. The empirically retrieved gain and spontaneous emission was used to predict the carrier distribution among dots. Results were compared with the modeled results and a good agreement with the random distribution of carrier among dot was observed. As 
an application of the proposed methodology, towards optimization of a QD laser device depending upon the carrier distribution followed was discussed.

\section{ACKNOWLEDGEMENT}

The author would like to thank University of Engineering \& Technology, Lahore, Pakistan, for their support in writing this paper.

\section{REFERENCES}

[1] Greenwood, P.D.L., Kennedy, K., Groom, K.M., Hugues, M., Hopkinson, M., Hogg, R.A., Krstajicì, N., Smith, L.E., Matcher, S.J., Bonesi, M., MacNeil, S., and Smallwood, R., "Quantum Dot Super Luminescent Diodes for Optical Coherence Tomography: Device Engineering”, IEEE Journal of Selected Topics in Quantum Electronics, Volume 16, No. 4, pp. 1015-1022, UK, 2010.

[2] Rafailov, E.U., Cataluna, M.A., and Sibbett, W., "ModeLocked Quantum-Dot Lasers", Nature Photonics, Volume 1, pp. 395-401, UK, 2007.

Stevens, B.J., Childs, D.T.D., Shahid, H., and Hogg, R.A., "Direct Modulation of Excited State Quantum Dot Lasers", Applied Physics Letters, Volume 95, No. 6, pp. 61101, UK, 2009.

[4] Grundmann, M., and Bimberg, D., "Theory of Random Population for Quantum Dots”, Physical Review-B, Volume 55, No. 15, pp. 9740, Germany, 1997.

[5] Summers, H.D., Thomson, J.D., Smowton, P.M., Blood, P., and Hopkinson, M., "Thermodynamic Balance in Quantum Dot Lasers", Semiconductor Science Technology, Volume 16, pp. 140-143, UK, 2001.

[6] Spencer, P., Clarke, E., Howe, P., and Murray, R., "Inhomogeneous Gain Effects in Quantum Dot Lasers", Electronic Letters, Volume 43, No. 10, pp. 574-575, UK, 2007.
[7] O'Driscoll, I., Blood, P., and Smowton, P.M., "Random Population of Quantum Dots in InAs-GaAs Laser Structures", IEEE Journal of Quantum Electron, Volume 46, No. 4, pp. 525-532, UK, 2010.

[8] Goldmann, E., Lorke, M., Frauenheim, Y., and Jahnke, F., "Negative Differential Gain in Quantum Dot Systems: Interplay of Structural Properties and Many-Body Effects", Applied Physics Letters, Volume 104, pp. 242108, Germany, 2014.

Avrutin, E.A., Marsh, J.H., and Portnoi, E.L., "Monolithic and Multi-Giga Hertz Mode-Locked Semiconductor Lasers: Constructions, Experiments, Models and Applications", IEE Proceedings of Optoelectronics, Volume 147, No. 4, pp. 251-278, UK, 2000.

[10] Finch, P., Blood, P., Smowton, P.M., Sobiesierski, A., Gwilliam, R.M., and O’Driscoll, I., "Femtosecond Pulse Generation from a Two-Section Mode-Locked QuantumDot Laser Using Random Population”, Proceedings of SPIE, Novel In-Plane Semiconductor Lasers, Volume XIII, pp. 90020E, UK, 2014.

[11] Hutchings, M., O’Driscoll, I., Smowton, P.M., and Blood, P., "Fermi-Dirac and Random Carrier Distributions in Quantum Dot Lasers", Applied Physics Letters, Volume 104, pp. 31103, UK, 2014.

[12] Otsubo, K., Hatori, N., Ishida, M., Okumura, S., Akiyama, T., Nakata, Y., Ebe, H., Sugawara M., and Arakawa, Y., "Temperature-Insensitive Eye-Opening under 10-Gb/s Modulation of 1.3- $\mu \mathrm{m}$ P-Doped Quantum-Dot Lasers without Current Adjustments", Japanese Journal Applied Physics, Volume 43, pp. 1124-1126, Japan, 2004.

[13] Shahid, H., Childs, D.T.D., Stevens, B.J., and Hogg, R.A., "Comparison of Gain Measurement Techniques for 1.3ìm Quantum Dot Lasers", Novel In-Plane Semiconductor Lasers, Proceedings of SPIE, pp. 79531W, UK, 2011. 
[14] Shahid, H., Childs, D.T.D., Stevens, B.J., and Hogg, R.A., "Negative Differential Gain Due to Many Body Effects in Self-Assembled Quantum Dot Lasers", Applied Physics Letters, Volume 99, pp. 61104, UK, 2011.

[15] Hakki, B.W., and Paoli, T.L., "Continuous Wave Degradation at $300 \mathrm{~K}$ of GaAs Double Heterostructure Junction Lasers-II. Electronic Gain", Journal of Applied Physics, Volume 44, pp. 4113-4119, USA, 1973.

[16] Bimberg, D., Grundmann, M., and Ledentsov, N.N., "Quantum Dot Heterostructures", Wiley, Germany, 1999.
[17] Schneider, H.C., Chow, W.W., and Koch, S.W., "ManyBody Effects in the Gain Spectra of Highly Excited Quantum-Dot Lasers", Physics Review-B, Volume 64, No. 11, pp. 115315, New Mexico, 2001.

[18] Majid, M.A., Chen, S.C., Childs, D.T.D., Shahid, H., Airey, R.J., Kennedy, K., Hogg, R.A., Clarke, E., Spencer, P., and Murray, R., "Gain and Absorption Characteristics of Bilayer Quantum Dot Lasers Beyond 1.3ìm”, Novel In-Plane Semiconductor Lasers-X, Proceedings of SPIE, pp. 795303, UK, 2011. 